Pacific Journal of Mathematics

THE REGULARITY OF MINIMAL SURFACES DEFINED OVER 


\section{THE REGULARITY OF MINIMAL SURFACES DEFINED OVER SLIT DOMAINS}

\section{DAVID KINDERLEHRER}

Let $\Omega$ denote the disc $x_{1}^{2}+x_{2}^{2}<r^{2}$ in the $x=\left(x_{1}, x_{2}\right)$ plane from which the segment $\left\{0 \leqq x_{1}<r, x_{2}=0\right\}$ has been deleted. Suppose that $u(x) \in C^{0}(\bar{\Omega})$ is a solution to the minimal surface equation in $\Omega((1)$ below) and attains boundary values $f\left(x_{1}\right) \in C^{1, \alpha}(0<\alpha<1)$ on the slit $\left\{0 \leqq x_{1}<r, x_{2}=0\right\}$. We shall prove here that the gradient of $u, D u=\left(u_{x_{1}}, u_{x_{2}}\right)$, is continuous at the origin $x=0$.

There is a corresponding result for harmonic functions, due to H. Lewy [7], which we paraphrase here. If $u(x) \in C^{0}(\bar{\Omega})$ is harmonic and attains boundary values $f\left(x_{1}\right) \in C^{1, \alpha}(0<\alpha<1)$ on the slit $\left\{0 \leqq x_{1}<r, x_{2}=0\right\}$, then

$$
\liminf _{h \uparrow 0} \frac{1}{h}(u(h, 0)-u(0,0))=\left\{\begin{array}{l}
\infty, \text { or } \\
\left\{\begin{array}{l}
-\infty \\
f^{\prime}(0)
\end{array}\right.
\end{array}\right.
$$

When the last alternative holds, $D u(x)$ is continuous at $x=0$. The harmonics $u_{ \pm}(x)= \pm \rho^{1 / 2} \sin \theta / 2, x=\rho e^{i \theta}$, illustrate the occurrence of the $\infty$ and $-\infty$ as possible limit values. The result to be proven here is, then, another example of the greater regularity possessed by solutions of the minimal surface equation (cf Bers [2], Nitsche [9], and [4]).

As an application, we consider the problem of minimizing the non-parametric area integrand among functions constrained to lie above a given function defined on a segment in a domain. More precisely, let $P$ be a bounded, open, convex domain with smooth boundary, $\sigma$ a closed straight segment in $P$, and $f(x)$ a continuous nonnegative convex function on $\sigma$ which vanishes at the endpoints of $\sigma$. Denote by

$$
\mathscr{K}=\left\{v(x) \in C^{0,1}(\bar{P}): v(x) \geqq f(x) \text { on } \sigma \text { and } v=0 \text { on } \partial P\right\} .
$$

The problem is then

(A) Prove that there exists a $u(x) \in \mathscr{K}$ such that

$$
\int_{P} \int \sqrt{1+|D u(x)|^{2}} d x=\min _{v \in \mathfrak{x}} \int_{p} \int \sqrt{1+|D v(x)|^{2}} d x .
$$

Evidently, a solution to $A$, if it exists, satisfies (1) in the set 
$\{x \in P: u(x)>f(x)\}$. Johannes C. C. Nitsche [10], considering, in fact, a larger class of surfaces than $\mathscr{K}$ above has proven:

(B) If $P$ is symmetric with respect to a line and $\sigma$ lies on this line of symmetry, then there exists a solution to $A$.

Furthermore, he has shown:

(C) If a solution to $A$ exists, it is unique. Moreover the set $\tau=\{x \in P: u(x)=f(x)\}$ is a (connected) sub-interval of $\sigma$.

Using the theorem to be proved here in addition to some similar elementary considerations, we may prove

Theorem I. If $u(x)$ is a solution to $A$ where $f \in C^{1, \alpha}(\sigma), 0<\alpha<1$, then $\partial u / \partial x_{1}$ is continuous in $\bar{P}$ and $\partial u / \partial x_{2}$ is continuous in $\bar{P}-\tau$ and upon one-sided approach to $\tau$. In addition $\left|\partial u / \partial x_{1}\right|$ is bounded by a constant depending only on $P, \sigma$, and $f$.

For the solution of $B$, Nitsche has shown the second part of Theorem $I([10]$, p. 105). We remark briefly on the proof of Theorem I at the conclusion of this paper. Primarily, we wish to prove

Theorem II. Let $u(x) \in C^{2}(\Omega) \cap C^{0}(\bar{\Omega})$ satisfy

$$
\begin{aligned}
\left(1+u_{x_{2}}^{2}\right) u_{x_{1} x_{1}}-2 u_{x_{1}} u_{x_{2}} u_{x_{1} x_{2}}+\left(1+u_{x_{1}}^{2}\right) u_{x_{2} x_{2}} & =0 \text { in } \Omega \\
u\left(x_{1}, 0\right) & =f\left(x_{1}\right), 0 \leqq x_{1}<r,
\end{aligned}
$$

where $f\left(x_{1}\right) \in C^{1, \alpha}([0, r]), 0<\alpha<1$.

Then $D u(x)$ is continuous at $x=0$.

To prove Theorem II, we shall utilize known properties of the conformal representation of the surface

$$
S=\left\{\left(x, x_{3}\right): x_{3}=u(x), x \in \Omega\right\}
$$

together with Lemma 1 below. In brief, $S$ may be viewed as a minimal surface whose boundary contains a spike. The boundary behavior of such surfaces is known. We quote here Theorems $D$ and $E$. To compute $u_{x_{1}}, u_{x_{2}}$ in terms of parameters $(\xi, \eta)$ different from $\left(x_{1}, x_{2}\right)$ involves the determination of three functional determinants, one of which, the Jacobian $J=\partial\left(x_{1}, x_{2}\right) / \partial(\xi, \eta)$, occurs as a denominator. The fact that $S$ has a one-to-one projection onto a slit domain is used to show that $J$ has "lowest order" among the three determinants.

We close with remarks about extensions to weaker boundary regularity.

2. The conformal representation and its properties. In this paragraph we introduce conformal parameters so that the minimal 
surface $S=\left\{\left(x, x_{3}\right): x_{3}=u(x), x \in \Omega\right\} \quad$ in $\left(x_{1}, x_{2}, x_{3}\right)$ space may be considered to be a minimal surface with a spike (cf [4]). We then determine regularity properties of this representation.

Denote by $G$ the open upper half $\zeta=\xi+i \eta$ plane. By a conformal representation of $S$ we shall understand a triple of harmonic functions.

$$
X(\zeta)=\left(x_{1}(\zeta), x_{2}(\zeta), x_{3}(\zeta)\right), \zeta \in G
$$

continuous in $\bar{G}$ and admitting finite limits at $\pm \infty$, which is a oneto-one map of $G$ onto $S$ and satisfies the isothermal relations

$$
X_{\xi}(\zeta)^{2}=X_{\eta}(\zeta)^{2} \text { and } X_{\xi}(\zeta) \cdot X_{\eta}(\zeta)=0, \zeta \in G \text {. }
$$

According to a result of Beckenbach and Rado [1], such a representation for $S$ exists because $u \in C^{0}(\bar{\Omega})$. We may assume that $X(0)=(0,0, f(0))$ and that the curve $x_{3}=f\left(x_{1}\right), x_{2}=0,0 \leqq x_{1}<r$, is the one-to-one continuous image of $-\xi_{1}<\xi \leqq 0$ and the one-to-one continuous image of $0 \leqq \xi<\xi_{2}$, for some $\xi_{1}, \xi_{2}>0$.

For the discussion which follows, it is more convenient to consider the conformal representation

$$
Y(\zeta)=\left(y_{1}(\zeta), y_{2}(\zeta), y_{3}(\zeta)\right), \zeta \in G
$$

obtained from $X(\zeta)$ above through the Euclidean motion

$$
\begin{aligned}
& y_{1}=x_{1} \cos \beta+\left(x_{3}-f(0)\right) \sin \beta \\
& y_{2}=x_{2} \\
& y_{3}=-x_{1} \sin \beta+\left(x_{3}-f(0)\right) \cos \beta,
\end{aligned}
$$

where

$$
\beta=\arctan f^{\prime}(0) \text {. }
$$

Note that $|\beta|<\pi / 2$. Evidently, $d y_{1} /\left.d x_{1}\right|_{x_{1}=0}>0$ and $d y_{3} /\left.d x_{1}\right|_{x_{1}=0}=0$ on the curve $x_{3}=f\left(x_{1}\right), x_{2}=0,0 \leqq x_{1}<r$.

After a conformal mapping of $G$ onto itself, if necessary, the conformal representation $Y(\zeta)$ satisfies these conditions:

$y_{1}(\xi)$ is strictly decreasing from $\bar{y}$ to 0 for $-1<\xi \leqq 0$

$y_{1}(\xi)$ is strictly increasing from 0 to $\bar{y}$ for $0 \leqq \xi<1$,

for some $\bar{y}>0$, and

$$
y_{2}(\xi)=0, y_{3}(\xi)=g\left(y_{1}(\xi)\right) \text { for }|\xi|<1
$$

where $g\left(y_{1}\right)$ is the $C^{1, \alpha}$ function of $y_{1}$ obtained by setting $x_{3}=f\left(x_{1}\right)$.

The conformal representation $Y(\zeta)$ is a representation of $S$ as a minimal surface with the spike

$$
\Gamma: y_{3}=g\left(y_{1}\right), y_{2}=0,0 \leqq y_{1}<\bar{y} ; g(0)=g^{\prime}(0)=0 \text {. }
$$


Let $F_{j}(\zeta)=y_{j}(\zeta)+i y_{j}^{*}(\zeta)$, where $y_{j}^{*}(\zeta)$ denotes the harmonic conjugate to $y_{j}(\zeta), F_{j}(0)=0, j=1,2,3$. It is well known, [12], that $F_{j}(\zeta)$ have absolutely continuous boundary values for $\operatorname{Im} \zeta=0$. About the $F_{j}(\zeta)$ we state Theorems $D$ and $E$ which are Theorem 1 [4] together with its corollary and Theorem $4^{\prime}$ [5] respectively.

THEOREM D. There is a neighborhood $U=\{|\zeta|<R, \operatorname{Im} \zeta>0\}$ and $a$ branch of $z=F_{1}(\zeta)^{1 / m}, m>0$ even integer, such that $z=F_{1}(\zeta)^{1 / m}$ is a univalent map of $U$ onto a domain in the (ordinary) $z=x+i y$ plane.

The curve $\gamma$ which is the image of $[-1,1] \cap \bar{U}$ under this mapping meets at a straight angle at $z=0$. Its tangent has a modulus of continuity proportional to $g^{\prime}\left(y_{1}\right)$ at $z=0$.

THEOREM E. There is a neighborhood $U=\{|\zeta|<R, \operatorname{Im} \zeta>0\}$ such that

$$
F_{1}(\zeta)^{1 / m}, F_{j}(\zeta) \in C^{1, \alpha}(\bar{U}), j=2,3,
$$

where $m>0$, even, is the integer determined in Theorem $D$.

For the proof of $E$, we refer to Theorem 4 in [5]. In addition to the facts just quoted, we require

Lemma 1. The functions $F_{\jmath}^{\prime}$ admit the expansions

$$
F_{j}^{\prime}(\zeta)=a_{j} \zeta+b_{j}(\zeta), \zeta \in \bar{U}, j=1,2,3
$$

where $a_{1}$ is real, $a_{2}, a_{3}$ are imaginary, $\left|a_{1}\right| \geqq\left|a_{2}\right|>0$ and $\left|b_{j}(\zeta)\right| \leqq C|\zeta|^{1+\alpha}$ for $\zeta \in \bar{U}, C>0$, a constant.

The asymptotic expansion of the $F_{j}^{\prime}(\zeta)$ provided by Theorem $E$, and stated explicitly in Lemma 1, is similar to those in [11], which is for minimal surfaces, and [3] which is for surfaces satisfying certain assumptions about their mean curvature. Both of these require the boundary to be of class $C^{2}$ and "regular," although the constants corresponding to $a_{j}$ and $C$ above depend only a priori on the given data. However, the existence of the tangent plane to a minimal surface when the boundary is suitably smooth has been known for some time [8].

3. Proof of Theorem II assuming Lemma 1. In terms of the given $\left(x_{1}, x_{2}, x_{3}\right)$ coordinates, the mapping $\zeta \rightarrow x(\zeta)=\left(x_{1}(\zeta), x_{2}(\zeta)\right)$ is a one-to-one harmonic mapping. In view of (2), its Jacobian is 


$$
\begin{aligned}
J= & \operatorname{Im}\left(F_{1}^{\prime}(\zeta) \cos \beta-F_{3}^{\prime}(\zeta) \sin \beta\right) \overline{F_{2}^{\prime}(\zeta)} \\
= & i a_{1} a_{2} \cos \beta|\zeta|^{2}+\operatorname{Im}\left\{a_{1} \zeta \overline{b_{2}(\zeta)}+\bar{a}_{2}^{\bar{\zeta}} b_{1}(\zeta)+b_{1}(\zeta) \overline{\left.b_{2}(\zeta)\right\}} \cos \beta\right. \\
& -\operatorname{Im}\left\{a_{3} \zeta \overline{b_{2}(\zeta)}+\bar{a}_{2} \bar{\zeta} b_{3}(\zeta)+b_{3}(\zeta) \overline{\left.b_{2}(\zeta)\right\}} \sin \beta\right. \\
= & i a_{1} a_{2} \cos \beta|\zeta|^{2}+B_{1}(\zeta), \zeta \in \bar{U} .
\end{aligned}
$$

Here we have used that $a_{1}$ is real and $a_{2}, a_{3}$ are imaginary. After two similar computations, we find that

$$
\frac{\partial\left(x_{2}, x_{3}\right)}{\partial(\xi, \eta)}=-i a_{1} a_{2} \sin \beta|\zeta|^{2}+B_{2}(\zeta), \zeta \in \bar{U}
$$

and

$$
\frac{\partial\left(x_{1}, x_{3}\right)}{\partial(\xi, \eta)}=i a_{1} a_{3}|\zeta|^{2}+B_{3}(\zeta), \zeta \in \bar{U}
$$

The $B_{j}(\zeta)$ satisfy $\left|B_{j}(\zeta)\right| \leqq C|\zeta|^{2+\alpha}$ for a constant $C>0$.

Therefore, for $x$ in the image of $\bar{U}$ under $x(\zeta)$,

$$
\frac{\partial u}{\partial x_{1}}(x)=f^{\prime}(0)+R(\zeta), \text { where }|R(\zeta)| \leqq \text { const. }|\zeta|^{\alpha} .
$$

But an elementary computation reveals that $x_{1}^{2}+x_{2}^{2} \geqq$ const. $|\zeta|^{4}$, for $|\zeta|$ sufficiently small. Hence

$$
\left|\frac{\partial u}{\partial x_{1}}-f^{\prime}(0)\right| \leqq \text { const }|x|^{\alpha / 2} \text { for } x \in \bar{\Omega},|x|
$$

sufficiently small. In the same way

$$
\left|\frac{\partial u}{\partial x_{2}}-\frac{1}{\cos \beta} \frac{a_{3}}{a_{2}}\right| \leqq \text { const }|x|^{\alpha / 2} \text { for } x \in \bar{\Omega},
$$

$|x|$ sufficiently small. Here we have used the abbreviation "const." to denote a positive constant, not necessarily the same at each occurrence.

The question of determining an a priori limitation of $\left(\partial u / \partial x_{2}\right)(0)$ is different in nature, and will be considered elsewhere.

4. Proof of Lemma 1. The proof of Lemma 1 is divided into the two lemmas below. Note that the strict monotonicity of $y_{1}(\xi)$ in $-1<\xi \leqq 0$ and $0 \leqq \xi<1$ implies the existence of continuous functions $H_{j}\left(y_{1}\right), j=1,2$, such that $y_{1}^{*}(\xi)=H_{1}\left(y_{1}(\xi)\right)$ for $-1<\xi \leqq 0$ and $y_{1}^{*}(\xi)=H_{2}\left(y_{1}(\xi)\right)$ for $0 \leqq \xi<1$.

LEMMA 2. (a) $H_{j}\left(y_{1}\right)$ are absolutely continuous functions of $y_{1}$ and $\left|H_{j}^{\prime}\left(y_{1}\right)\right| \leqq C_{1}\left|g^{\prime}\left(y_{1}\right)\right|$, a.e., $0 \leqq y_{1} \leqq \bar{y}, C_{1}>0$ constant.

(b) $\lim _{\xi \rightarrow 0}\left|\frac{\partial y_{2}^{*}}{\partial \xi}(\xi)\left(\frac{\partial y_{1}}{\partial \xi}(\xi)\right)^{-1}\right| \leqq 1$ 
(c) $\left|F_{j}^{\prime}(\xi)\right| \leqq C_{2}\left|F_{1}^{\prime}(\xi)\right| \leqq C_{3}|\xi|^{m-1} \quad$ for $\quad|\xi|<1, \xi \in \bar{U}, j=2,3$, where $m \geqq 2$ is the integer determined in Theorem $D$ and $C_{2}, C_{3}>0$ are constants. $U$ is the set of Theorem $E$.

Proof. Let $s$ denote the arc length of the minimal surface on $\Gamma: y_{3}=g\left(y_{1}\right), y_{2}=0,0 \leqq y_{1} \leqq \bar{y}$. According to Tsuji's result [12],

$$
0 \neq\left(\frac{d s}{d \xi}\right)^{2}=\left(1+g^{\prime}\left(y_{1}\right)^{2}\right)\left(\partial y_{1} / \partial \xi\right)^{2} \text {, a.e. for }|\xi|<1 \text {. }
$$

Therefore, $\partial y_{1} / \partial \xi \neq 0$ a.e. for $-1<\xi<1$. It follows that the inverse function $\xi=h\left(y_{1}\right)$ to $y_{1}(\xi)$ on $-1<\xi \leqq 0$ is absolutely continuous for $0 \leqq y \leqq \bar{y}$. Since $h$ is also monotone, $H_{1}\left(y_{1}\right)=y_{1}^{*}\left(h\left(y_{1}\right)\right)$ is absolutely continuous for $0 \leqq y \leqq \bar{y}$.

Furthermore,

$$
\left(\frac{d s}{d \xi}\right)^{2}=\sum_{1}^{3}\left(\frac{\partial y_{j}^{*}}{\partial \xi}\right)^{2} \text { for }|\xi|<1 .
$$

Hence for a constant $\mathrm{C}_{1}>0$,

$$
\sum_{1}^{3}\left(\frac{y_{j \xi}^{*}}{y_{1 \xi}}\right)^{2} \leqq \sup \left(1+g^{\prime}\left(y_{1}\right)^{2}\right)=C_{1}^{2} \text { for }|\xi|<1 .
$$

Using the isothermal relation

$$
\sum_{1}^{3} y_{j \xi}(\xi) y_{j \xi}^{*}(\xi)=0,|\xi|<1,
$$

we obtain that

$$
H_{1}^{\prime}\left(y_{1}\right)=-g^{\prime}\left(y_{1}\right) \frac{\partial y_{3}^{*}}{\partial \xi}\left(\frac{\partial y_{1}}{\partial \xi}\right)^{-1} \text {, a.e. for }-1 \leqq \xi \leqq 0
$$

Hence

$$
\left|H_{1}^{\prime}\left(y_{1}\right)\right| \leqq C_{1}\left|g^{\prime}\left(y_{1}\right)\right| \text { a.e., }-1 \leqq \xi \leqq 0 .
$$

Now from (3),

$$
\left(y_{2 \xi}^{*}(\xi)\right)^{2} \leqq\left(1+g^{\prime}\left(y_{1}\right)^{2}\right) y_{1 \xi}(\xi)^{2},|\xi|<1 .
$$

Hence (b) follows.

Finally

$$
\left|F_{j}^{\prime}(\xi)\right|^{2} \leqq \sum_{1}^{3}\left|F_{j}^{\prime}(\xi)\right|^{2}=2\left(\frac{d s}{d \xi}\right)^{2} \leqq 2\left(1+g^{\prime}\left(y_{1}\right)^{2}\right)\left|F_{1}^{\prime}(\xi)\right|^{2}
$$

which implies that

$$
\left|F_{j}^{\prime}(\xi)\right| \leqq \sqrt{2} C_{1}\left|F_{1}^{\prime}(\xi)\right| \text { for }|\xi|<1 .
$$


Now $F_{1}(\zeta)^{1 / m} \in C^{1, \alpha}(\bar{U})$, for a suitable $U$, by Theorem $E$; hence,

$$
F_{1}(\xi)=\frac{1}{m} a_{1} \xi^{m}+A_{1}(\xi)
$$

and $F_{1}^{\prime \prime}(\xi)=a_{1} \xi^{m-1}+b_{1}(\xi),|\xi|<1$ and $\xi \in \bar{U},\left|b_{1}(\xi)\right| \leqq$ const. $|\xi|^{m-1+\alpha}$ and $a_{1} \neq 0$. That $a_{1} \neq 0$ is insured by the existence of a tangent with a suitable modulus of continuity to the curve $\gamma: z=F_{1}(\xi)^{1 / m}, \xi \in \bar{U}$, (cf Theorem $D$ ). Also, $\left|F_{1}^{\prime}(\xi)\right| \leqq$ const. $|\xi|^{m-1}, \xi \in \bar{U}$, from which (c) follows.

Lemma $3 . \quad F_{2}(\zeta)$ admits the representation

$$
F_{2}(\zeta)=\frac{1}{2} a_{2} \zeta^{2}+\sum_{k>2} c_{k} \zeta^{k},|\zeta|<1
$$

where $a_{2} \neq 0, c_{k}$ are imaginary.

\section{Also the integer $m=2$.}

Proof. Since $\operatorname{Re} F_{2}(\xi)=y_{2}(\xi)=x_{2}(\xi)=0$ for $|\xi|<1, F_{2}$ admits a development as that above, perhaps with a linear term, with $a_{2}, c_{k}$ imaginary. We must demonstrate that $a_{2} \neq 0$ and $c_{1}=0$. This follows from a well known argument about harmonic mappings [2]. The mapping $\zeta \rightarrow\left(x_{1}(\zeta), y_{2}(\zeta)\right)$ is a one-to-one harmonic map. Hence by a lemma of Lewy [6], $\partial\left(x_{1}, y_{2}\right) / \partial(\xi, \eta) \neq 0$ in $|\zeta|<1, \operatorname{Im} \zeta>0$, and therefore $F_{2}^{\prime}(\zeta) \neq 0$ in $|\zeta|<1, \operatorname{Im} \zeta>0$. For $\lambda$ real, we consider the inverse image

$$
C=\left\{|\zeta|<1, \operatorname{Im} \zeta>0: y_{2}(\zeta)=\lambda\right\}
$$

of $y_{2}=\lambda$ in $\Omega$. If not empty, $C$ is an analytic curve in $\operatorname{Im} \zeta>0$, $|\zeta|<1$ since $\zeta \rightarrow\left(x_{1}, y_{2}\right)$ is an analytic homeomorphism whose Jacobian does not vanish. For $\zeta \in C$,

$$
F_{2}^{\prime}(\zeta)=\left(\frac{\partial y_{2}}{\partial t}+i \frac{\partial y_{2}^{*}}{\partial t}\right)\left(\frac{d \zeta}{d t}\right)^{-1} \neq 0
$$

where $t$ denotes the tangent direction on $C$. Hence $d y_{2}^{*} / d t \neq 0$ on $C$, so that $F_{2}(\zeta)$ is monotone on $C$. Hence $F_{2}$ is univalent in $|\zeta|<1$, $\operatorname{Im} \zeta>0$, from which it follows that

$$
F_{2}(\zeta)=\frac{1}{n} a_{n} \zeta^{n}+\sum_{n>n} c_{k} \zeta^{k}, \text { with } a_{n} \neq 0, n \leqq 2 .
$$

By the previous lemma

$$
\left|F_{2}^{\prime}(\xi)\right| \leqq c_{1}\left|F_{1}^{\prime}(\xi)\right| \leqq c_{2}|\xi|^{m-1}, m \geqq 2 \text { even } .
$$


Therefore $2 \geqq n \geqq m \geqq 2$ or $m=n=2$.

Proof of Lemma 1. Since $m=2$, we know that

$$
F_{j}^{\prime}(\zeta)=a_{j} \zeta+b_{j}(\zeta), \zeta \in \bar{U}, \text { with }\left|b_{j}(\zeta)\right| \leqq C|\zeta|^{1+\alpha}
$$

for $j=1,2,3$. By Lemma 2(b) and Lemma 3,

$$
\left|a_{1}\right| \geqq\left|\operatorname{Re} a_{1}\right| \geqq\left|a_{2}\right|>0 \text {. }
$$

It remains to show that $a_{1}$ is real and $a_{3}$ is imaginary. Using Lemma $2(a)$,

$$
H_{1}^{\prime}\left(y_{1}\right)=\frac{\operatorname{Im} F_{1}^{\prime}(\xi)}{\operatorname{Re} F_{1}^{\prime}(\xi)}=\frac{\operatorname{Im} a_{1}+\operatorname{Im} b_{1}(\xi) \xi^{-1}}{\operatorname{Re} a_{1}+\operatorname{Re} b_{1}(\xi) \xi^{-1}}, \xi<0,
$$

and $\left|H_{1}^{\prime}\left(y_{1}\right)\right| \leqq C_{1}\left|g^{\prime}\left(y_{1}\right)\right| \rightarrow 0$ as $y_{1} \rightarrow 0$. Hence $\operatorname{Im} a_{1}=0$. Now according to the isothermal relations

$$
\sum F_{j}^{\prime}(\zeta)^{2}=0
$$

hence $a_{1}^{2}+a_{2}^{2}+a_{3}^{2}=0$. Since $a_{1}$ is real, $a_{2}$ is imaginary, and $\left|a_{1}\right| \geqq\left|a_{2}\right|$, the relation implies that $\left(a_{3}\right)^{2} \leqq 0$. Hence $a_{3}$ is imaginary.

We wish to remark here that by assuming only that $f^{\prime}\left(x_{1}\right)$ satisfies $\int_{0}^{a} t^{-1}\left|f^{\prime}(t)\right| d t<\infty$, some $a>0$, it is possible to prove that $\partial u / \partial x_{1}$ is continuous as $x \rightarrow 0$ in any sector $0<\tau \leqq \arg x \leqq 2 \pi-\tau$. The proof is by the same argument, except that Theorem $E$ must be replaced by a fact analogous to the existence of the angular derivative as proved by S. Warschawski [13]. This fact, whose proof requires a generalization of a classical theorem of Lindelöf, is not difficult to prove.

We now remark briefly on the proof of Theorem I. The technique by which continuity of $D u(x)$ was shown at the end points of the segment $\tau$ in Theorem II may be utilized in a simpler fashion to show that $u_{x_{1}}(x)$ and $u_{x_{2}}(x)$ are continuous at each interior point of $\tau$. Continuity of $u_{x_{2}}(x)$ is understood to mean continuity upon one-sided approach to $\tau$. In fact, the functions analogous to $F_{j}^{\prime}(\zeta)$ in Lemma 1 admit an expansion of the form " $a_{j}+b_{j}(\zeta)$ " with $\left|b_{j}(\zeta)\right| \leqq c|\zeta|^{\alpha}$, suitable $c>0$, where the $a_{j}$ satisfy the conclusions of Lemma 1 .

Given $x^{0} \in \partial P, D u\left(x^{0}\right)$ may be estimated by the slopes of the plane tangent to the space curve $\partial P$ at $\left(x_{1}^{2}, x_{2}^{2}, 0\right)$ and some point of the curve $x_{3}=f\left(x_{1}\right), x_{2}=0$. This estimate depends only on the given data. Finally, we observe that $u_{x_{1}}(x)$ satisfies a maximum principle in $P-\tau$. Hence $\sup _{\bar{P}}\left|u_{x_{1}}(x)\right| \leqq \max \left(\sup _{\partial P}|D u(x)|, \sup \left|f^{\prime}\left(x_{1}\right)\right|\right)$. 


\section{REFERENCES}

1. E. F. Beckenbach and Tibor Rado, Subharmonic functions and minimal surfaces, Trans. Amer. Math. Soc., 35 (1933), 648-661.

2. L. Bers, Isolated singularities of minimal surfaces, Annals of Math., 53.2 (1951), $364-386$.

3. E. Heinz, Uber das Randverhalten quasilinear elliptischer Systeme, Math. Zeit., 13.2 (1970), p. 99.

4. D. Kinderlehrer, Minimal surfaces whose boundaries contain spikes, J. Math. and Mech., 19.9, (1970), 829-853.

5. - The boundary regularity of minimal surfaces, Annali della S.N.S. di Pisa, 23.4, (1969), 711-744.

6. H. Lewy, On the nonvanishing of the Jacobian in certain one-to-one mappings, Bull. Amer. Math. Soc., 42 (1936), 689-692.

7. - On a variational problem with inequalities on the boundary, J. Math. Mech., 17 (1968), 861-884.

8. - On the analytic continuation of minimal surfaces and similar problems, Proceedings of the fourth international congress of mathematicians, vol. II (1961) p. 233 (In Russian; published in 1964).

9. J. C. C. Nitsche, On new results in the theory of minimal surfaces, Bull. Amer. Math. Soc., 71, (1965), 195-270.

10. - Variational problems with inequalities as boundary conditions, or how to fashion a cheap hat for Giacometti's brother, Archive for Rat. Mech. and Anal., 35.2, (1969), 83-113.

11. - The boundary behavior of minimal surfaces-Kellogg's theorem and branch points on the boundary, Invent. Math., 8 (1969).

12. M. Tsuji, On a theorem of $F$ and $M$ Riesz, Proc. Imp. Acad. Tokyo 18 (1942), $172-175$.

13. S. Warschawski, On the differentiability at the bounday in conformal mapping, Proc. Amer. Math. Soc., 12 (1961), 614-620.

Received February 26, 1970. This research was supported in part by Grant AFOSR-883-67.

UNiversity of MinNesota 



\section{PACIFIC JOURNAL OF MATHEMATICS}

\section{EDITORS}

H. SAMELSON

Stanford University

Stanford, California 94305

C. R. Новву

University of Washington

Seattle, Washington 98105
J. DUGundJI

Department of Mathematics

University of Southern California

Los Angeles, California 90007

RICHARD ARENS

University of California

Los Angeles, California 90024

\section{ASSOCIATE EDITORS}
E. F. BECKENBACH
B. H. NeumanN
F. WOLF
K. YOSHIDA

\section{SUPPORTING INSTITUTIONS}
UNIVERSITY OF BRITISH COLUMBIA
CALIFORNIA INSTITUTE OF TECHNOLOGY
UNIVERSITY OF CALIFORNIA
MONTANA STATE UNIVERSITY
UNIVERSITY OF NEVADA
NEW MEXICO STATE UNIVERSITY
OREGON STATE UNIVERSITY
UNIVERSITY OF OREGON
OSAKA UNIVERSITY
UNIVERSITY OF SOUTHERN CALIFORNIA
STANFORD UNIVERSITY
UNIVERSITY OF TOKYO
UNIVERSITY OF UTAH
WASHINGTON STATE UNIVERSITY
UNIVERSITY OF WASHINGTON
AMERICAN MATHEMATICAL SOCIETY CHEVRON RESEARCH CORPORATION TRW SYSTEMS
NAVAL WEAPONS CENTER 


\section{Pacific Journal of Mathematics}

\section{Vol. 37, No. $1 \quad$ January, 1971}

Gregory Frank Bachelis and Haskell Paul Rosenthal, On unconditionally

converging series and biorthogonal systems in a Banach space .........

Richard William Beals, On spectral theory and scattering for elliptic

operators with singular potentials .........................

J. Lennart (John) Berggren, Solvable and supersolvable groups in which every element is conjugate to its inverse ........................ 21

Lindsay Nathan Childs, On covering spaces and Galois extensions ..........

William Jay Davis, David William Dean and Ivan Singer, Multipliers and

unconditional convergence of biorthogonal expansions..............

Leroy John Derr, Triangular matrices with the isoclinal property ............

Paul Erdős, Robert James McEliece and Herbert Taylor, Ramsey bounds for

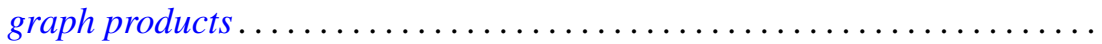

Edward Graham Evans, Jr., On epimorphisms to finitely generated

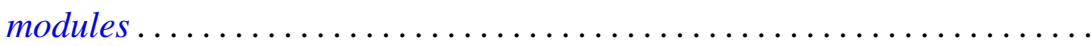

Hector O. Fattorini, The abstract Goursat problem ................. 51

Robert Dutton Fray and David Paul Roselle, Weighted lattice paths .........

Thomas L. Goulding and Augusto H. Ortiz, Structure of semiprime $(p, q)$

radicals ...........................................

E. W. Johnson and J. P. Lediaev, Structure of Noether lattices with join-principal maximal elements ....

David Samuel Kinderlehrer, The regularity of minimal surfaces defined over

slit domains

Alistair H. Lachlan, The transcendental rank of a theory. .

Frank David Lesley, Differentiability of minimal surfaces at the boundary ...

Wolfgang Liebert, Characterization of the endomorphism rings of divisible torsion modules and reduced complete torsion-free modules over complete discrete valuation rings....

Lawrence Carlton Moore, Strictly increasing Riesz norms.

Raymond Moos Redheffer, An inequality for the Hilbert transform ...

James Ted Rogers Jr., Mapping solenoids onto strongly self-entwined,

circle-like continua..........................

Sherman K. Stein, B-sets and planar maps ................... 217

Darrell R. Turnidge, Torsion theories and rings of quotients of Morita

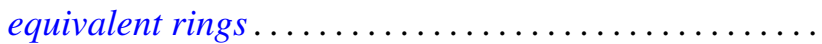

Fred Ustina, The Hausdorff means of double Fourier series and the principle of localization ................................

Stanley Joseph Wertheimer, Quasi-compactness and decompositions for arbitrary relations.

Howard Henry Wicke and John Mays Worrell Jr., On the open continuous images of paracompact $\check{C}$ ech complete spaces... 